\title{
Siting and sizing of distributed generators via NSGA-II and max-min composition
}

\author{
Andrei C. Ribeiro ${ }^{1}$, Francisco V. E. Lemos ${ }^{1}$, Fábio R. Barbosa ${ }^{2}$ \\ ${ }^{1}$ Estudante do Programa de Pós-graduação em Engenharia Elétrica \\ Universidade Federal do Piauí (UFPI) \\ Teresina - PI - Brasil \\ ${ }^{2}$ Professor do Departamento de Engenharia Elétrica \\ Universidade Federal do Piauí (UFPI) \\ Teresina - PI - Brasil \\ \{andrei, victorlemos, fabiorocha\}@ufpi.edu.br
}

\begin{abstract}
Distributed Generation (DG) can provide a series of benefits, with loss reduction being the most important. Although this generation reaches increasing levels of penetration, the associated costs are still high. This paper presents a multiobjective optimization model for sizing and siting of DG. NSGA-II was used as a search tool to minimize the active power losses and to reduce the installation, operation and maintenance costs. The simulations were performed in MATPOWER considering four versions of the 69-bus system. The max-min composition was used for trade-off and final choice at the Pareto frontier. The tools used provided superior results to other works in the literature.
\end{abstract}

Resumo. A Geração Distribuída (GD) pode proporcionar uma série de benefícios, sendo a redução das perdas o mais importante. Porém, mesmo com essa geração alcançando níveis crescentes de penetração, os custos associados ainda são elevados. Este trabalho apresenta um modelo de otimização multiobjetivo para o dimensionamento e posicionamento de geradores. Utilizou-se o NSGA-II como ferramenta de busca para minimizar as perdas de potência ativa e reduzir o custo anualizado. As simulações foram realizadas no MATPOWER em quatro versões do sistema de 69 barras. A composição max-min foi utilizada para trade-off e escolha final na fronteira de Pareto. As ferramentas utilizadas proporcionaram resultados superiores a outros trabalhos da literatura.

\section{Introdução}

Atualmente, principalmente devido à legislação propícia [ANEEL 2012, ANEEL 2015, ANEEL 2017], há uma forte tendência no país para a redução da distância entre a produção e o consumo de energia elétrica, através da Geração Distribuída (GD) [EPE 2018]. Esse tipo de geração, também conhecida como geração embarcada e geração descentralizada, possibilita gerar energia elétrica através de pequenas centrais geradoras, geralmente estimuladas por recursos renováveis, localizadas próximo aos consumidores finais [Ackermann et al. 2001].

A GD ganha destaque por ser uma solução economicamente viável e ambientalmente adequada para expansão. Apesar de empreendimentos dessa natureza apresentarem 
um custo de instalação superior a outras opções, observa-se uma redução contínua nesse valor graças aos avanços tecnológicos alcançados [Bansal 2017]. A geração descentralizada também apresenta impactos ambientais menores do que as usinas de potência tradicionais que dependem da queima de combustíveis fósseis [Akella et al. 2009].

Com a inserção adequada de geradores distribuídos, o sistema pode se tornar mais robusto e confiável. Por exemplo, a performance pode ser melhorada com a redução das perdas de potência nas linhas e um perfil de tensão mais homogêneo nas barras dentro de limites de operação [Guedes 2006]. Porém, caso os geradores não sejam posicionados e dimensionados de forma adequada, a GD pode comprometer a operação do sistema [Pepermans et al. 2005, Guedes 2006].

Este trabalho apresenta um modelo multiobjetivo e sua solução para o problema de alocação ótima de GD, definindo a posição e dimensionamento de geradores distribuídos em redes de distribuição radiais. O modelo tem como objetivos reduzir as perdas de potência ativa e minimizar o custo de instalação, operação e manutenção sob certas restrições e premissas.

A solução é determinada a partir de algoritmos genéticos (NSGA-II) e lógica Fuzzy (composição max-min). A composição max-min é usada para atender o compromisso de escolha final de uma solução da fronteira de Pareto.

\section{Modelagem}

As funções-objetivo ou funções de avaliação são de extrema importância por estabelecerem uma conexão entre um problema do mundo real e um modelo proposto para solução. Duas funções-objetivo são apresentadas para avaliar a qualidade das alocações.

A primeira função é minimizar as perdas ativas. Essa função é o principal impacto técnico desejado com a inserção de GD em redes de distribuição radiais, sendo representado conforme a Equação 1:

$$
\min f_{1}(\bar{X})=\sum_{i=1}^{N_{r}} \frac{\left|\frac{v_{f}}{\tau e^{j \theta} \text { shift }}-v_{t}\right|^{2}}{r_{s}-j x_{s}}
$$

As perdas são calculadas com auxílio do MATPOWER [Zimmerman et al. 2010], ferramenta responsável pelo cálculo do fluxo de potência.

Embora avaliar as perdas de potência ativa seja a principal métrica para a performance de uma rede, a sua minimização não deve ser o único objetivo considerado. O segundo objetivo proposto é baseado na modelagem econômica apresentada em [Buayai et al. 2012], o custo de investimento total de um gerador distribuído $k$ é proporcional ao produto do custo unitário $U C_{k}$ pela injeção máxima de potência $C_{k, \max }^{G D}$.

Esse custo é transformado em um custo anualizado, Equação 2, a partir de um fator anualizado $A F_{i}$ como mostra a Equação 3:

$$
\begin{gathered}
\min f_{2}(\bar{X})=\sum_{k=1}^{N_{G D}}\left(A F_{i} \cdot U C_{k} \cdot C_{k, \max }^{G D}\right) \\
A F_{i}=\frac{(i / 100)(1+i / 100)^{T}}{(1+i / 100)^{T}-1}
\end{gathered}
$$


onde, $A F_{i}$ é o fator anualizado mencionado, que transforma o custo do empreendimento em custo anual baseado em determinada taxa de juros $i$ e vida útil $T$. A taxa de juros de $6,5 \%$ ao ano e um horizonte de estudo de 20 anos foram escolhidos, respectivamente, para $i$ e $T$. Além disso, definiu-se $U C_{k}$ igual a $4.600 \mathrm{R} \$ / \mathrm{kW}$.

As seguintes restrições foram adotadas para dar factibilidade às soluções. A geração total, isto é, geração centralizada e geração distribuída a ser incluída, deve ser igual a soma das perdas com a demanda das cargas. Essa restrição (de igualdade) conhecida como balanço de potência ativa e reativa é representada pela Equação 4:

$$
\left\{\begin{array}{l}
\sum_{i=1}^{N_{b}} P_{G}=P_{L}+\sum P_{D} \\
\sum_{i=1}^{N_{b}} Q_{G}=Q_{L}+\sum Q_{D}
\end{array}\right.
$$

Segundo o Módulo 8 do PRODIST [ANEEL 2018], em sistemas com tensão de referência superior a $1 \mathrm{kV}$ e inferior a $69 \mathrm{kV}$, o limite inferior de tensão $V_{\min }$ é igual a $93 \%$ e o limite superior $V_{\max }$ é igual a $105 \%$ da tensão nominal $V_{\text {ref }}$.

$$
\underbrace{0,93 V_{\text {ref }}}_{V_{\min }} \leq V_{n} \leq \underbrace{1,05 V_{\text {ref }}}_{V_{\max }}
$$

Considerando a Resolução Normativa $n^{\circ}$ 687/2015 [ANEEL 2015], a potência instalada máxima por alocação $S_{G D}$ será igual a $5 \mathrm{MW}$. Enquanto, a quantidade de geradores inseridos $N_{G D}$ foi fixada em 4 unidades. Essa restrição é um dos principais gargalos para algoritmos de busca exaustiva. Além disso, o valor de $N_{G D}$ tende a aumentar a medida que sistemas maiores são considerados.

Segue então o modelo completo na Equação 6, contendo o conjunto de equações e inequações que representam o problema de otimização: minimizar as perdas ativas e o custo de instalação, operação e manutenção dadas as restrições de potência, tensão e projeto.

$$
\min \left\{\begin{array} { l } 
{ f _ { 1 } ( \overline { X } ) = \sum _ { i = 1 } ^ { N _ { r } } \frac { | \frac { v _ { f } } { \tau e ^ { j \theta _ { s } \text { sift } } } - v _ { t } } { r _ { s } - j x _ { s } } | ^ { 2 } } \\
{ f _ { 2 } ( \overline { X } ) = \sum _ { k = 1 } ^ { N _ { G D } } ( A F _ { i } \cdot U C _ { k } \cdot C _ { k , \text { max } } ^ { G D } ) }
\end{array} \text { s.t. } \left\{\begin{array}{l}
\sum_{i=1}^{N_{b}} P_{G}=P_{L}+\sum P_{D} \\
\sum_{i=1}^{N_{b}} Q_{G}=Q_{L}+\sum Q_{D} \\
0,93 V_{\text {ref }} \leq V_{n} \leq 1,05 V_{\text {ref }} \\
N_{G D}=4 \\
S_{G D} \leq 5 M W
\end{array}\right.\right.
$$

Outras premissas relevantes foram introduzidas ao modelo proposto:

1. Todas as barras, exceto a barra 1 (slack bus), são candidatas à alocação de GD.

2. Um barramento não pode receber mais de uma alocação.

3. Geradores distribuídos são modelados como cargas negativas.

4. A carga do sistema é considerada PQ constante.

5. A carga não varia ao longo do horizonte de estudo.

6. Todas as barras, exceto a barra 1, são rotuladas com um perfil de carga: industrial, comercial e residencial.

7. O sistema apresenta, conforme a Tabela 1, quatro versões de patamares de carga, leve, média, pesada e pico. 
Tabela 1. Coeficientes atribuídos aos patamares de carga leve, média, pesada e pico, considerando as barras com perfil residencial, comercial e industrial.

\begin{tabular}{ccc}
\hline \hline Patamar de carga & Perfil de carga & Coeficiente de carga \\
\hline \hline \multirow{2}{*}{ Leve } & Residencial & 0,4 \\
& Comercial & 0,4 \\
& Industrial & 1,0 \\
\hline \multirow{3}{*}{ Média } & Residencial & 1,0 \\
& Comercial & 1,0 \\
& Industrial & 1,0 \\
\hline \multirow{3}{*}{ Pesada } & Residencial & 1,4 \\
& Comercial & 0,7 \\
& Industrial & 0,7 \\
\hline \multirow{3}{*}{ Pico } & Residencial & 1,4 \\
& Comercial & 1,0 \\
& Industrial & 1,0 \\
\hline \hline
\end{tabular}

\section{Estratégia de solução}

A estratégia implementada para otimização do modelo é apresentada. O método de busca e a técnica integrada à busca para compromisso de escolha final são mostrados nas duas subseções a seguir.

\subsection{NSGA-II}

O Non-Dominated Sorting Genetic Algorithm-II (NSGA-II) é um algoritmo evolucionário de otimização multiobjetivo. Ele foi criado para suprir as carências e críticas da versão anterior [Deb et al. 2002].

Na Figura 1 é resumido o funcionamento do NSGA-II. Esse algoritmo constrói uma população $R_{i}$ com os pais $P_{i}$ e filhos $Q_{i}$. Em seguida, cria fronteiras $F_{1}, F_{2}, F_{3}$ e assim sucessivamente com os indivíduos, de acordo com o nível de não-dominância.

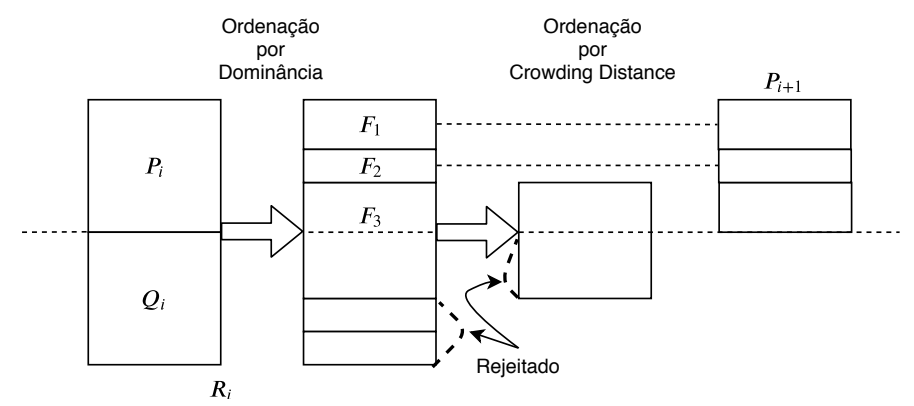

Figura 1. Fluxograma que mostra como o NSGA-II funciona. $P_{i}$ é a população de pais e $Q_{i}$ a de filhos na geração $i$. $F_{1}$ engloba as melhores soluções da população combinada (pais e filhos). $F_{2}$ são as melhores soluções excluindo $F_{1}$ e assim sucessivamente.

A técnica de seleção adotada é o torneio binário. O crossover intermediário, também conhecido como crossover aritmético, foi escolhido como operador de busca 
local. Esse operador utiliza o material genético de dois vetores-pais para produzir os vetores-filhos a partir de uma média ponderada por $a$, como descrito na Equação (7).

$$
\left\{\begin{array}{l}
\text { Filho }_{1}=a \cdot \text { Pai }_{1}+(1-a) \cdot \text { Pai }_{2} \\
\text { Filho }_{2}=(1-a) \cdot \text { Pai }_{1}+a \cdot \text { Pai }_{2}
\end{array}\right.
$$

A mutação gaussiana também conhecida como mutação normal foi adotada como operador de busca global. Esse operador é definido pela Equação (8) e (9).

$$
\begin{gathered}
\text { Filho }=\text { Pai }+S * \operatorname{Rand} *\left(U_{b}-L_{b}\right) \\
S=\text { Scale } *(1-\operatorname{shrink} * \text { Curr } G / \text { Max } G)
\end{gathered}
$$

Onde $S$ determina o desvio padrão do número aleatório gerado Rand. É importante destacar também que conforme o progresso da otimização continua, o shrink diminui o intervalo de mutação. As outras variáveis $U_{b}, L_{b}, \operatorname{Curr} G$ e $M a x G$ representam o limite superior, o limite inferior, a geração atual e a geração máxima, respectivamente. Por fim, na Tabela 2 os parâmetros utilizados no NSGA-II são resumidos.

Tabela 2. Parâmetros utilizados no NSGA-Il para alocação de GD.

\begin{tabular}{lc}
\hline \hline NSGA-II (parâmetro) & Valor do parâmetro \\
\hline \hline Tamanho da população $-N_{\text {pop }}$ & 50 \\
Número de gerações - MaxG & 100 \\
Coeficiente de ponderação do crossover - $a$ & 1,25 \\
Limite inferior de Siting $-L_{b}$ & 2 \\
Limite superior de Siting $-U_{b}$ & 69 \\
Limite inferior de Sizing $-L_{b}$ & 0 \\
Limite superior de Sizing $-U_{b}$ & 5 \\
\hline \hline
\end{tabular}

\subsection{Fuzzy max-min}

A abordagem proposta em [Cano 2007] foi adaptada para atender ao compromisso de escolher uma solução entre as que formam a fronteira de Pareto na última geração do algoritmo de busca.

O uso de métodos de satisfação difusa com objetivos antagônicos é comum. Como o modelo proposto é formado por dois objetivos conflitantes, soluções extremas irão sempre priorizar um objetivo, enquanto rejeitam outro. Por exemplo, soluções que apresentam as menores perdas, possuem um alto custo associado. Dessa forma, é interessante que se adote uma região de saturação onde a função de pertinência para as perdas $\mu_{\alpha}$ será máxima.

$$
\mu_{\alpha}=\left\{\begin{array}{l}
1, \text { para } \alpha_{i} \leq \alpha_{\min } \\
\frac{\alpha_{\max }-\alpha_{i}}{\alpha_{\max }-\alpha_{\min }}, \text { para } \alpha_{\min }<\alpha_{i}<\alpha_{\max } \\
0, \text { para } \alpha_{i} \geq \alpha_{\max }
\end{array}\right.
$$

Adotou-se o valor de $\alpha_{\min }=0,2$ e $\alpha_{\max }=1,0$. A escolha desse parâmetro, assim como $\beta_{\text {min }}$, não é arbitrária, esses valores foram definidos observando as avaliações dos indivíduos na última população. 
Da mesma forma, definiu-se uma região de saturação onde a função de pertinência para o custo $\mu_{\beta}$ será constante.

$$
\mu_{\beta}=\left\{\begin{array}{l}
1, \text { para } \beta_{i} \leq \beta_{\min } \\
\frac{\beta_{\max }-\beta_{i}}{\beta_{\max }-\beta_{\min }}, \text { para } \beta_{\min }<\beta_{i}<\beta_{\max } \\
0, \text { para } \beta_{i} \geq \beta_{\max }
\end{array}\right.
$$

Adotou-se o valor de $\beta_{\min }=0,05$ e $\beta_{\max }=1,0$ para o sistema de 69 barras.

\section{Resultados}

Na Figura 2 está representado o sistema de 69 barras e 68 ramos em 12,66 kV proposto em [Das 2008]. O perfil de carga foi distribuído em faixas assim como em [Hung et al. 2014]. Portanto, adotou-se o trecho das barras 2 até a 27 e as barras 47, 48, 49, 50, 51, 52, 66, 67,68 , e 69 com o perfil residencial, a faixa da barra 28 até a 46 com perfil comercial e as barras restantes com perfil industrial.

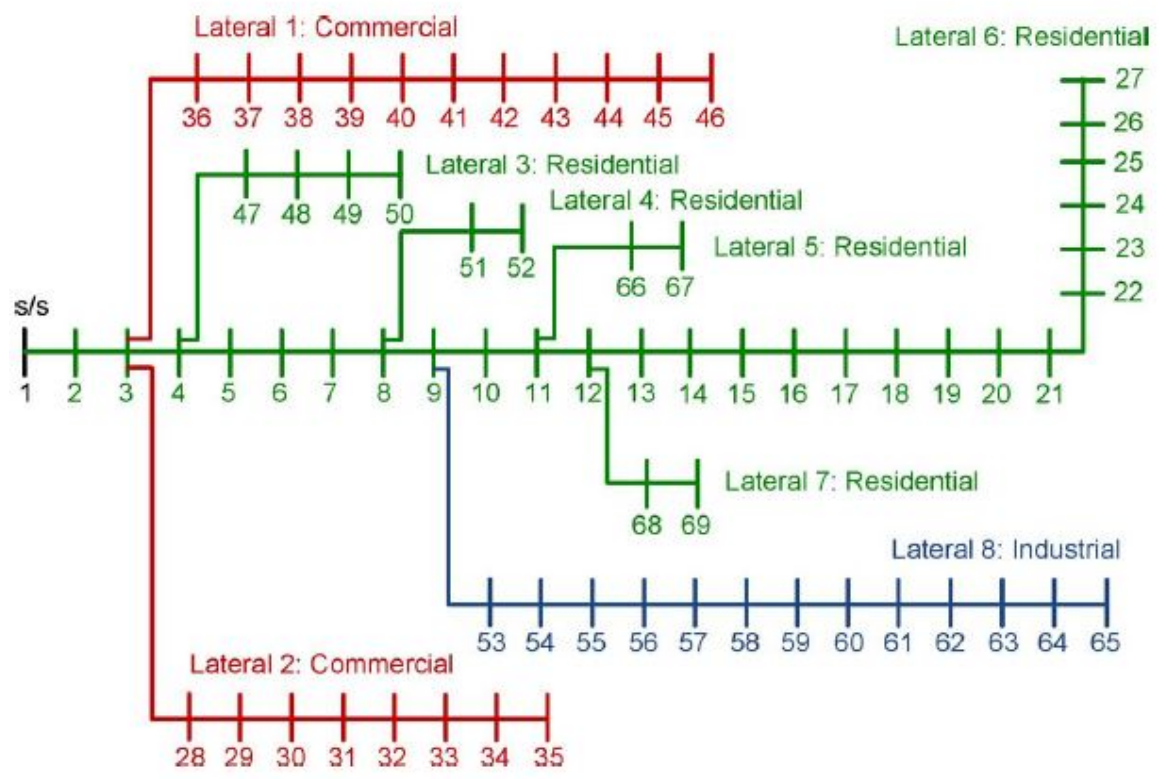

Figura 2. Sistema de distribuição radial com 69 barras.

No cenário de carga leve, o sistema de 69 barras apresentou uma potência instalada de $S=2,6 \mathrm{MW}+$ j1,8 MVAr, com 185,4 kW de perdas, equivalente a 7,14\% da geração. No cenário de carga média, apresentou uma potência instalada de $S=3,8 \mathrm{MW}+\mathrm{j} 2,7$ MVAr, com 225,0 kW de perdas ou igual 5,0\%. No patamar de carga pesada, $\mathrm{S}=3,9$ $\mathrm{MW}+\mathrm{j} 2,8 \mathrm{MVAr}$, com $154,1 \mathrm{~kW}$ de perdas ou igual a 4,9\%. No patamar de pico de carga, $\mathrm{S}=4,51 \mathrm{MW}+\mathrm{j} 3,20 \mathrm{MVAr}$, com $261,0 \mathrm{~kW}$ de perdas ou igual a 4,81\%.

Os perfis de tensão do sistema para os quatro patamares de carga considerados pré-alocação são apresentados na Figura 3. Os limites regulatórios, 0,93 p.u. $(11,77$ kV) e 1,05 p.u. (13,29 kV), são ilustrados por linhas horizontais. Foram observadas violações de tensão nos patamares de carga leve, média e pico.

A centésima geração do algoritmo de busca é ilustrada na Figura 4. Nela também são destacadas as soluções escolhidas pela composição max-min em cada patamar de 


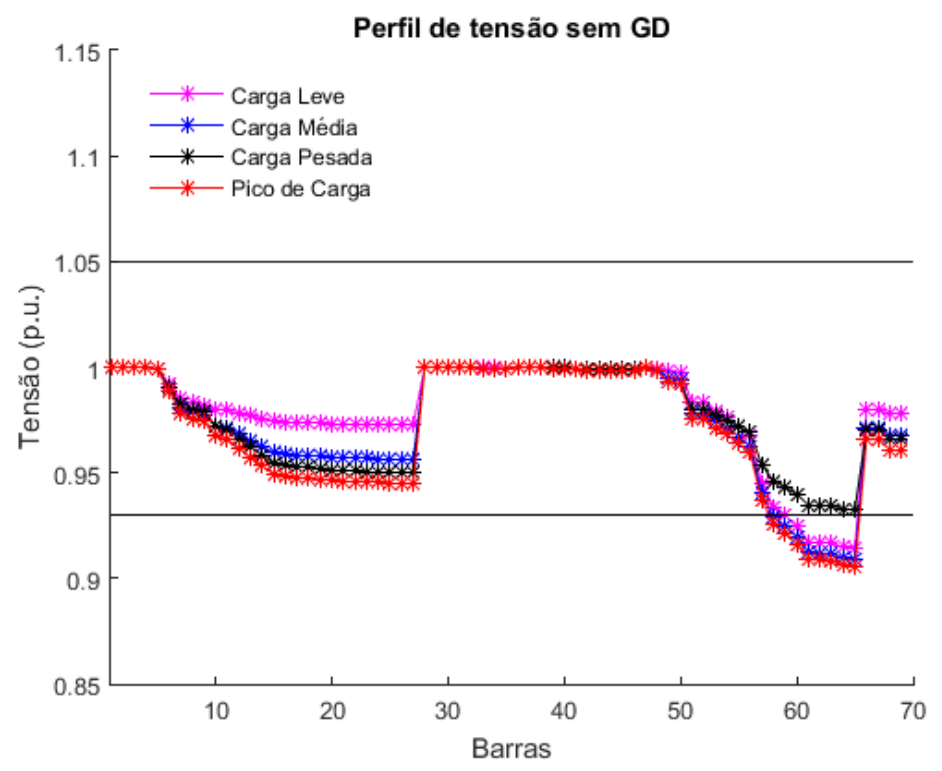

Figura 3. Curva de perfil de tensão para o sistema de 69 barras nos patamares de carga leve, média, pesada e pico sem a presença de geradores distribuídos.

carga. A solução escolhida para o patamar de carga leve (solução 1) apresenta um tradeoff equilibrado entre os objetivos, especialmente pela performance de seu caso referência. Os outros patamares exigiram soluções cuja alocação apresentava índice de penetração maior e priorizava a redução de perdas.

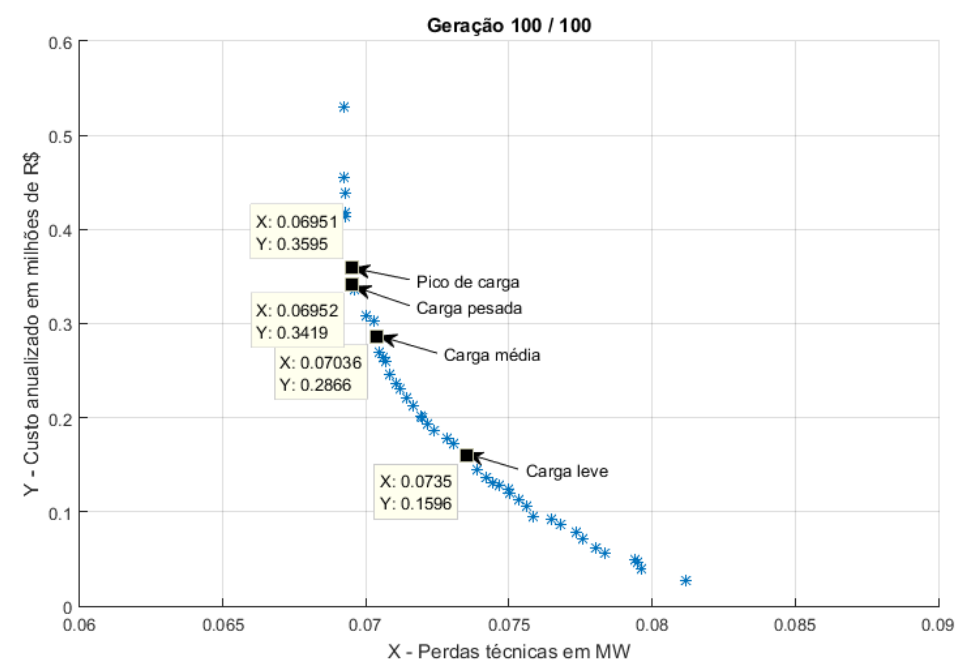

Figura 4. Curva de perfil de tensão para o sistema de 69 barras nos patamares de carga leve, média, pesada e pico sem a presença de geradores distribuídos.

Na Figura 5, a convergência do NSGA-II é ilustrada a partir do valor médio e do menor valor por geração alcançado nos dois objetivos.

As quatro soluções por patamar de carga estão apresentadas na Tabela 3. Como 


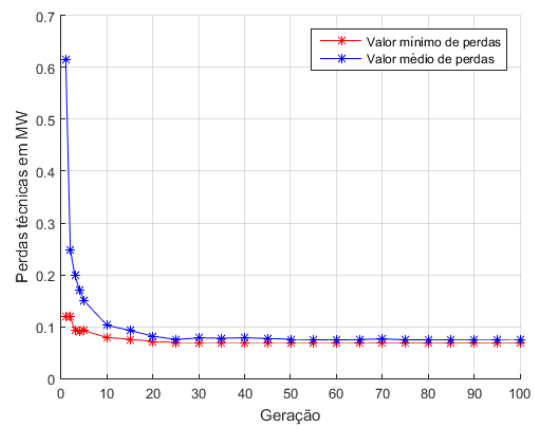

(a) Convergência para perdas

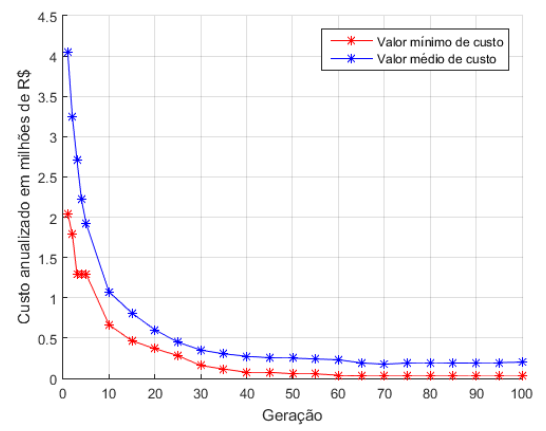

(b) Convergência para custo

Figura 5. Curvas de convergência do NSGA-II para alocação de GD no sistema de 69 barras

o custo anualizado da alocação $f_{2}$ independe da versão do sistema, apenas o objetivo $f_{1}$ é recalculado nos outros patamares, o impacto de cada solução nos dois objetivos é apresentado na Tabela 4.

Tabela 3. Barras candidatas à alocação e tamanho dos geradores distribuídos para cada patamar de carga através de composição max-min.

\begin{tabular}{lllllllll}
\hline \hline Patamar de carga & \multicolumn{4}{c}{ Siting } & \multicolumn{5}{c}{ Sizing $(M W)$} \\
\hline \hline Leve (solução 1) & 21 & 61 & 64 & 69 & 0.1311 & 0.1228 & 0.0358 & 0.0889 \\
Média (solução 2) & 21 & 61 & 64 & 69 & 0.2077 & 0.1228 & 0.0343 & 0.3150 \\
Pesada (solução 3) & 11 & 12 & 21 & 61 & 0.0281 & 0.1179 & 0.2031 & 0.4617 \\
Pico (solução 4) & 11 & 12 & 21 & 61 & 0.0151 & 0.1179 & 0.2579 & 0.4617 \\
\hline
\end{tabular}

Tabela 4. Cenários sem alocação e pós-alocação considerando as soluções para cada patamar de carga no sistema de 69 barras.

\begin{tabular}{lccccc}
\hline \hline Patamar de carga & \multicolumn{2}{c}{ Leve } & Média & Pesada & Pico \\
\hline \hline Cenário & $f_{1}$ & $f_{2}$ & $f_{1}$ & $f_{1}$ & $f_{1}$ \\
sem GD & 0.1854 & 0 & 0.2250 & 0.1541 & 0.2608 \\
solução 1 & 0.0560 & 0.1562 & 0.0735 & 0.0601 & 0.0917 \\
solução 2 & 0.0571 & 0.2805 & 0.0704 & 0.0545 & 0.0856 \\
solução 3 & 0.0566 & 0.3345 & 0.0695 & 0.0525 & 0.0829 \\
solução 4 & 0.0572 & 0.3518 & 0.0695 & 0.0521 & 0.0824 \\
\hline \hline
\end{tabular}

Também, pode-se comparar, por patamar de carga, os cenários pré-alocação e pósalocação no sistema de 69 barras na Figura 6. Além do benefício técnico de redução das perdas, obteve-se um perfil de tensão mais homogêneo com a operação dentro dos limites especificados.

Na Tabela 5, as soluções alcançadas em trabalhos anteriores são listadas. A partir da comparação dos valores dos dois objetivos no patamar de carga média, o método proposto apresentou qualidade superior. 
Tabela 5. Qualidade das soluções propostas em outros trabalhos vs solução encontrada no método proposto para o patamar de carga média.

\begin{tabular}{|c|c|c|c|c|}
\hline Sistema & Trabalho & Solução (siting, sizing) & Perdas & Custo \\
\hline 69 barras & Esse trabalho & $\begin{array}{l}\text { barra } 21,0.2077 \mathrm{MW} \\
\text { barra } 61,0.1228 \mathrm{MW} \\
\text { barra } 64,0.0343 \mathrm{MW} \\
\text { barra } 69,0.3150 \mathrm{MW}\end{array}$ & $70.4 \mathrm{~kW}$ & 0.2805 \\
\hline 69 barras & [Prakash and Lakshminarayana 2016] & $\begin{array}{l}\text { barra } 11,0.5010 \mathrm{MW} \\
\text { barra } 18,0.4820 \mathrm{MW} \\
\text { barra } 61,1.7700 \mathrm{MW}\end{array}$ & $129.2 \mathrm{~kW}$ & 1.1358 \\
\hline 69 barras & [Nawaz et al. 2016] & $\begin{array}{l}\text { barra } 61,1.3300 \mathrm{MW} \\
\text { barra } 64,0.3100 \mathrm{MW} \\
\text { barra } 27,0.2100 \mathrm{MW}\end{array}$ & $128.2 \mathrm{~kW}$ & 0.7633 \\
\hline 69 barras & [Murty and Kumar 2015] & barra $61,1.8700 \mathrm{MW}$ & $128.1 \mathrm{~kW}$ & 0.7455 \\
\hline 69 barras & [Rao et al. 2012] & $\begin{array}{l}\text { barra } 65,0.1018 \mathrm{MW} \\
\text { barra } 64,0.3690 \mathrm{MW} \\
\text { barra } 63,1.3024 \mathrm{MW}\end{array}$ & $90 \mathrm{~kW}$ & 0.7316 \\
\hline 69 barras & [Acharya et al. 2006] & barra $61,1.8100 \mathrm{MW}$ & $128.3 \mathrm{~kW}$ & 0.7468 \\
\hline 69 barras & [Raut and Mishra 2019] & $\begin{array}{l}\text { barra } 63,0.1769 \mathrm{MW} \\
\text { barra } 61,0.3724 \mathrm{MW} \\
\text { barra } 62,0.1625 \mathrm{MW}\end{array}$ & $83.6 \mathrm{~kW}$ & 0.2937 \\
\hline
\end{tabular}

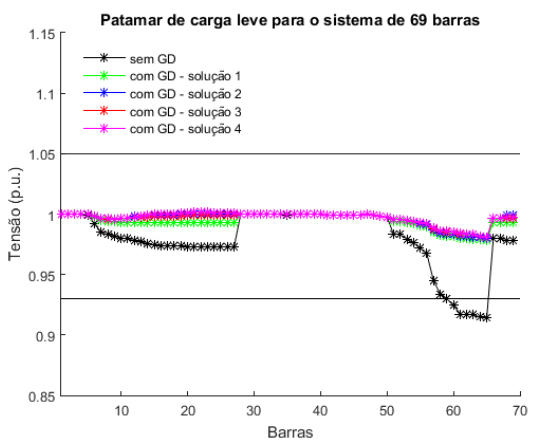

(a) Carga leve

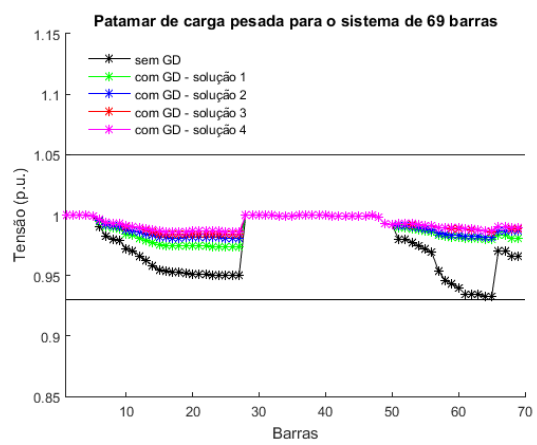

(c) Carga pesada

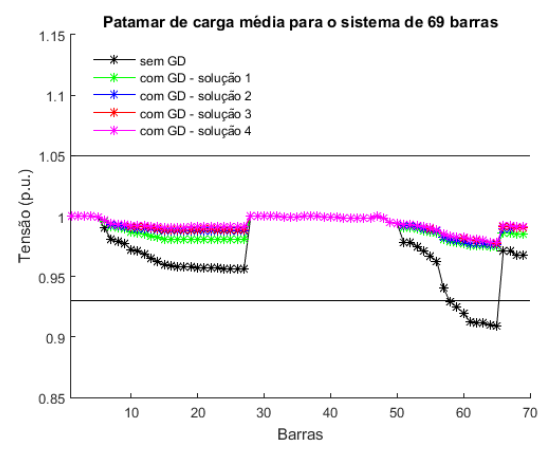

(b) Carga média

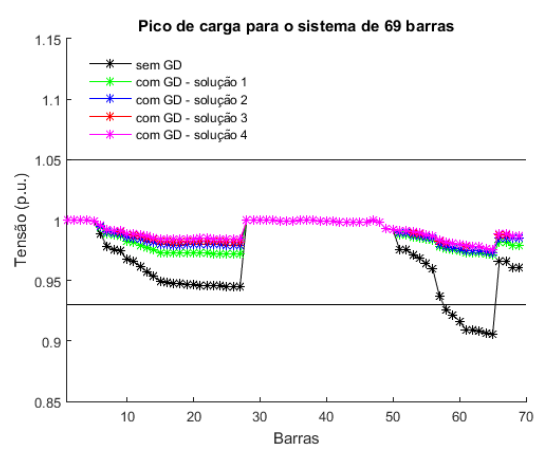

(d) Carga pico

Figura 6. Perfil de tensão no sistema de 69 barras no cenário sem GD e nos cenários com GD a partir das soluções escolhidas por patamar de carga. 


\section{Conclusão}

Sabe-se que o problema de alocação é um problema não linear de difícil tratamento, com a exploração total do espaço de busca sendo na maioria das vezes impraticável. Dessa forma, utilizar um método meta-heurístico como ferramenta de busca é uma escolha adequada. O método escolhido nesse trabalho foi o NSGA-II que apresentou uma boa convergência.

Além disso, por se tratar de um problema de otimização com objetivos conflitantes, não existirá uma solução ótima para todos os objetivos. Para compromisso de escolha, optou-se pela composição max-min como ferramenta de trade-off e seleção final. A composição implementada é tão simples quanto escolher um ponto intermediário da fronteira, porém mais versátil, pois permite a partir do ajuste dos coeficientes de pertinência adicionar informações e caracterizar a solução final às preferências desejadas.

Pode-se afirmar que com as inserções propostas, os resultados verificados foram satisfatórios, pois a redução dos objetivos foi alcançada e as restrições foram respeitadas. O impacto positivo das unidades de GD inseridas também pôde ser observado a partir dos perfis de tensão nos cenários pré-alocação e pós-alocação, com o valor de tensão nas barras mais próximo ao valor de referência.

É importante destacar que a superioridade alcançada com o método proposto considera apenas os objetivos, visto que é difícil comparar trabalhos diferentes, principalmente devido às singularidades de cada modelo (diferentes objetivos, restrições e premissas).

Para trabalhos futuros, outros algoritmos de otimização multiobjetivo, como o SPEA2 e o IBEA, serão utilizados. Além disso, resultados mais promissores são esperados com a substituição da sintonia manual dos coeficientes de pertinência da composição max-min por um mecanismo de inteligência computacional.

\section{Agradecimentos}

O presente trabalho foi realizado com apoio da Coordenação de Aperfeiçoamento de Pessoal de Nível Superior - Brasil (CAPES) - Código de Financiamento 001.

\section{Referências}

Acharya, N., Mahat, P., and Mithulananthan, N. (2006). An analytical approach for dg allocation in primary distribution network. International Journal of Electrical Power \& Energy Systems, 28(10):669-678.

Ackermann, T., Andersson, G., and Söder, L. (2001). Distributed generation: a definition. Electric power systems research, 57(3):195-204.

Akella, A., Saini, R., and Sharma, M. P. (2009). Social, economical and environmental impacts of renewable energy systems. Renewable Energy, 34(2):390-396.

ANEEL (2012). Resolução Normativa $n^{o} 482$ de 17 de abril de 2012. Agência Nacional de Energia Elétrica, Brasília.

ANEEL (2015). Resolução Normativa $n^{o} 687$ de 24 de novembro de 2015. Agência Nacional de Energia Elétrica, Brasília. 
ANEEL (2017). Resolução Normativa $n^{\circ} 786$ de 17 de outubro de 2017. Agência Nacional de Energia Elétrica, Brasília.

ANEEL (2018). Procedimentos de Distribuição de Energia Elétrica no Sistema Elétrico Nacional - Módulo 8. Agência Nacional de Energia Elétrica, Brasília.

Bansal, R. (2017). Handbook of distributed generation: electric power technologies, economics and environmental impacts. Springer.

Buayai, K., Ongsakul, W., and Mithulananthan, N. (2012). Multi-objective micro-grid planning by nsga-ii in primary distribution system. European Transactions on Electrical Power, 22(2):170-187.

Cano, E. B. (2007). Utilizing fuzzy optimization for distributed generation allocation. In TENCON 2007-2007 IEEE Region 10 Conference, pages 1-4. IEEE.

Das, D. (2008). Optimal placement of capacitors in radial distribution system using a fuzzy-ga method. International Journal of Electrical Power \& Energy Systems, 30(67):361-367.

Deb, K., Pratap, A., Agarwal, S., and Meyarivan, T. (2002). A fast and elitist multiobjective genetic algorithm: Nsga-ii. IEEE transactions on evolutionary computation, 6(2):182-197.

EPE (2018). Plano Decenal de Expansão de Energia 2027. Empresa de Pesquisa Energética, Brasília.

Guedes, L. d. M. (2006). Localização e dimensionamento de unidades de geração distribuída em redes de distribuição radiais. Master's thesis, Universidade de Brasília, Mestrado em Engenharia Elétrica.

Hung, D. Q., Mithulananthan, N., and Lee, K. Y. (2014). Determining pv penetration for distribution systems with time-varying load models. IEEE Transactions on Power Systems, 29(6):3048-3057.

Murty, V. and Kumar, A. (2015). Optimal placement of dg in radial distribution systems based on new voltage stability index under load growth. International Journal of Electrical Power \& Energy Systems, 69:246-256.

Nawaz, S., Bansal, A., and Sharma, M. (2016). An analytical approach for dg placement in reconfigured distribution networks. Journal of Applied Power Engineering, 5(3):137-143.

Pepermans, G., Driesen, J., Haeseldonckx, D., Belmans, R., and D'haeseleer, W. (2005). Distributed generation: definition, benefits and issues. Energy policy, 33(6):787-798.

Prakash, D. and Lakshminarayana, C. (2016). Multiple dg placements in distribution system for power loss reduction using pso algorithm. Procedia Technology, 25:785792.

Rao, R. S., Ravindra, K., Satish, K., and Narasimham, S. (2012). Power loss minimization in distribution system using network reconfiguration in the presence of distributed generation. IEEE transactions on power systems, 28(1):317-325. 
Raut, U. and Mishra, S. (2019). An improved elitist-jaya algorithm for simultaneous network reconfiguration and dg allocation in power distribution systems. Renewable Energy Focus, 30:92-106.

Zimmerman, R. D., Murillo-Sánchez, C. E., and Thomas, R. J. (2010). Matpower: Steady-state operations, planning, and analysis tools for power systems research and education. IEEE Transactions on power systems, 26(1):12-19. 